\title{
NIVELES DE RESISTENCIA A QUINOLONAS Y OTROS ANTIMICROBIANOS EN CEPAS DE Escherichia coli COMENSALES EN NIÑOS DE LA ZONA PERIURBANA DE LIMA, PERÚ
}

\author{
María J. Pons 1,a, Susan Mosquito 2,b, Theresa J. Ochoa ${ }^{2,3, c}$, Martha Vargas ${ }^{4, \mathrm{~d}}$, Margarita Molina ${ }^{5, \mathrm{e}}$, \\ Angela Lluque ${ }^{2, b}$, Ana I. Gil ${ }^{5, e}$, Lucie Ecker ${ }^{5, f}$, Francesca Barletta ${ }^{2, g}$, Claudio F. Lanata ${ }^{5,6, f}$, \\ Luis J. Del Valle ${ }^{7, d}$, Joaquim Ruiz ${ }^{1,8, d}$
}

\begin{abstract}
RESUMEN
El objetivo principal del estudio fue establecer el nivel de resistencia a antimicrobianos en un total de 222 cepas comensales de $E$. coli de origen fecal, en Perú. Las frecuencias de resistencia encontrados, frente los antimicrobianos evaluados, fueron: ampicilina (62,6\%), cotrimoxazol (48,6\%), tetraciclina $(43,0 \%)$ y cloranfenicol $(15,8 \%)$. Destacan los elevados niveles de resistencia a quinolonas: $32 \%$ al ácido nalidíxico (NAL) y $12 \%$ a ciprofloxacino (CIP). Estos elevados niveles hacia las quinolonas en cepas comensales aisladas en niños de esta franja de edad, realzan el uso extendido y el impacto de consumo de este tipo de antimicrobianos en la comunidad, mostrando el riesgo potencial de su pérdida de utilidad en el área.
\end{abstract}

Palabras clave: Pruebas de sensibilidad microbiana; Quinolonas; Escherichia coli; Bacterias fecales (fuente: DeCS BIREME).

\section{LEVELS OF QUINOLONES RESISTANCE AND OTHER ANTIMICROBIAL IN NON-PATHOGENIC Escherichia coli STRAINS IN CHILDREN FROM THE PERIURBAN AREA OF LIMA, PERU}

\begin{abstract}
The main aim of this study was to establish the resistance levels to antimicrobial agents, in 222 non-pathogenic E. Coli strains of fecal origin in Peru. The proportion of resistance found to the evaluated antimicrobials was ampicillin $(62.6 \%)$, cotrimoxazole $(48,6 \%)$, tetracycline $(43,0 \%)$ and chloramphenicol $(15,8 \%)$. We emphasize the high resistance levels found for quinolones: $32 \%$ for nalidixic acid (NAL) and 12\% for ciprofloxacin (CIP). These high levels of quinoloneresistance in non-pathogenic strains isolated from children in this age group highlight the extensive use and the impact of the intake of this kind of antimicrobials in the community, showing the potential risk of the loss of their utility in the area.
\end{abstract}

Key words: Microbial sensitivity tests; Quinolones; Escherichia coli; Fecal bacteria (source: MeSH NLM).

\section{INTRODUCCIÓN}

Desde la introducción de la penicilina en la práctica clínica, en la década de 1940, los antimicrobianos han sido usados para el tratamiento de enfermedades infecciosas. Desafortunadamente las bacterias han desarrollado resistencia a todos los antimicrobianos conocidos hasta el momento. Actualmente la resistencia a antimicrobianos, sobre todo la presencia de multirresistencia, es considerada uno de los problemas de salud de mayor relevancia a nivel mundial según la OMS ${ }^{(1)}$.

Las quinolonas son una familia de antimicrobianos que

\footnotetext{
Barcelona Centre fot International Health Researh (CRESIB, Hospital Clinic-Universitat de Barcelona). Barcelona, España.

Instituto de Medicina Tropical, Universidad Peruana Cayetano Heredia. Lima, Perú.

University of Texas School of Public Health. Houston, EE.UU.

Institut d'Investigacions Biomèdiques August Pi i Sunyer (IDIBAPS), Hospital Clínic. Barcelona, España.

Instituto de Investigación Nutricional. Lima, Perú

Escuela de Medicina, Universidad Peruana de Ciencias Aplicadas. Lima, Perú.

Departament d'Enginyeria Química, ETSEIB, Universitat Politècnica de Catalunya. Barcelona, España.

CIBER Epidemiología y Salud Pública (CIBERESP). Barcelona, España.

a Bióloga, magister en biomedicina; ${ }^{b}$ bióloga, magister en Microbiología; ${ }^{\mathrm{c}}$ médico, pediatra infectólogo; ${ }^{\mathrm{d}}$ biólogo, posdoctorado en biología; ${ }^{\mathrm{e}}$ microbióloga; ${ }^{\mathrm{f}}$ médico epidemiólogo; ${ }^{\mathrm{g}}$ bióloga molecular
}

Recibido: 03-11-11 Aprobado: 22-02-12 
fueron introducidos a la práctica clínica en 1967. Desde ese momento, debido a su amplio espectro de actividad, su uso se extendió rápidamente siendo actualmente uno de los antimicrobianos más prescritos a nivel mundial ${ }^{(2)}$.

Clásicamente estos antimicrobianos no han sido usados para tratar infecciones en niños, pero en informes recientes se muestra su seguridad en este grupo de edad (3). El mecanismo de resistencia más relevante frente a las quinolonas es la presencia de substituciones específicas en las dianas ${ }^{(2)}$, aunque, en los últimos 10 años se han descrito varios mecanismos transferibles de resistencia a quinolonas ${ }^{(4)}$.

La presencia de microorganismos comensales resistentes a antimicrobianos puede deberse a la presión directa del propio consumo de antimicrobianos o puede que, de manera alternativa, se deba a la adquisición de microorganismos resistentes. La Escherichia coli es un miembro típico de la microbiota intestinal, tanto en humanos como en animales ${ }^{(5)}$. Por ello, el análisis de los niveles de resistencia a antimicrobianos en cepas de $E$. coli comensales es un modelo válido para estimar el impacto del uso general de antimicrobianos en una área específica y una aproximación adecuada para predecir la evolución de la resistencia a quinolonas en la comunidad.

El objetivo del presente trabajo es analizar los niveles de resistencia a antimicrobianos en $E$. coli comensales aislados de niños peruanos de hasta doce meses.

\section{EL ESTUDIO}

Se realizó un estudio transversal de vigilancia de la diarrea infantil, donde se incluyeron muestras fecales recogidas entre octubre de 2006 y noviembre de 2007 de niños con diarrea $(\geq 3$ deposiciones líquidas 0 semilíquidas en menos de $24 \mathrm{~h} 0 \geq 1$ deposición con sangre) y controles (niños sanos que no hubiesen presentado diarrea una semana antes y una después de la recogida de la muestra) de 2 a 12 meses de edad ${ }^{(6)}$.

Para este subestudio de sensibilidad antimicrobiana, se utilizó las muestras de los niños sanos. Entraron en este grupo un total de 560 niños entre los 2 a 12 meses de edad, de los cuales se obtuvieron muestras fecales cada mes ${ }^{(6)}$.

De cada muestra de heces sembrada en medio Mc Conkey, se recuperaron cinco colonias lactosa positivas susceptibles de ser E. coli. Las colonias fueron identificadas por métodos bioquímicos convencionales y se descartó la presencia de E. coli diarrogénicas empleando real time PCR descrita en un estudio previo ${ }^{(6)}$, se seleccionó así las E.coli comensales. De todas aquellas muestras con $E$. coli comensales, se seleccionaron 222 cepas provenientes de 222 niños analizados en este estudio.

Se estableció la sensibilidad a ampicilina (AMP), amoxicilina más ácido clavulánico (AMC), cefotaxima (CTX), ceftazidima (CAZ), gentamicina (GEN), ácido nalidixico (NAL), ciprofloxacino (CIP), tetraciclina (TET), cloranfenicol (CHL), cotrimoxazol (SXT) y nitrofurantoina (NIT) mediante la técnica de difusión en disco, según la guía del Clinical and Laboratory and Standards Institute (CLSI) ${ }^{(7)}$. También se estableció la sensibilidad a azitromicina en 207 aislados usando la misma técnica.

La multirresistencia se definió como presencia de resistencia a tres o más agentes antimicrobianos no relacionados entre sí, excluyendo el caso de la azitromicina, debido a la falta de puntos de corte para Enterobacteriaceae ${ }^{(7)}$. Es por este mismo motivo por lo que la azitromicina se excluyó para las determinaciones de los perfiles de resistencia.

Se establecieron dos grupos para un mejor análisis de los resultados, un primer grupo con niños de edades comprendidas entre 0 y 6 meses y un segundo de mayores de 6 a 12 meses. Se usó la prueba de Fisher para el análisis estadístico de los resultados.

Se establecieron los diferentes fenotipos de sensibilidad a quinolonas, usando los puntos de corte recomendados por el CLSI, concretamente para NAL es resistente $\leq 13 \mathrm{~mm}$, intermedia $14-18$, sensible $\geq 19 \mathrm{~mm}$ y para CIP resistente $\leq 15 \mathrm{~mm}$, intermedia $16-18$, sensible $\geq$ $21 \mathrm{~mm}$.

Se describen los datos con frecuencias y porcentajes, procesados en una hoja de cálculo en Excel 2010.

Los niños escogidos entraron en el estudio después de que su responsable firmara el consentimiento informado. El estudio fue revisado y aprobado por el Comité de Ética del Instituto de Investigación Nutricional y de la Universidad Peruana Cayetano Heredia, Lima, Perú.

\section{HALLAZGOS}

En los resultados de sensibilidad obtenidos (Tabla 1), se observaron elevados niveles de resistencia a AMP $(62,6 \%)$, SXT $(48,6 \%)$ y TET $(43,0 \%)$, mientras que la resistencia fue menos elevada a $\mathrm{CHL}(15,8 \%)$ y GEN (10.4\%). Los niveles al resto de antimicrobianos testados fue menor al $10 \%$. 
Tabla 1. Niveles de resistencia a antimicrobianos en $E$. coli comensales, aislados de muestras fecales de niños sanos de Perú.

\begin{tabular}{|c|c|c|c|c|c|c|c|c|c|}
\hline \multirow[b]{2}{*}{ A.A } & \multicolumn{3}{|c|}{ Total de muestras $(\mathrm{N}=222)$} & \multicolumn{3}{|c|}{ Niños 0-6 meses $(\mathrm{N}=99)$} & \multicolumn{3}{|c|}{ Niños $7-12$ meses $(\mathrm{N}=123)$} \\
\hline & S & I & $\mathrm{R}$ & S & I & $\mathrm{R}$ & S & I & $\mathrm{R}$ \\
\hline AMP & $80(36,0)$ & $3(1,3)$ & $139(62,6)$ & $36(36,4)$ & $1(1,0)$ & $62(62,6)$ & $44(35,8)$ & $2(1,6)$ & $77(62,6)$ \\
\hline SXT & $114(51,4)$ & -- & $108(48,6)$ & $60(60,6)$ & -- & $39(39,4)$ & $54(43,9)$ & -- & $69(56,1) \dagger$ \\
\hline TET & $123(55,4)$ & $4(1,8)$ & $95(42,8)$ & $60(61,6)$ & $2(2,0)$ & $37(37,4)$ & $63(51,2)$ & $2(1,6)$ & $58(47,1)$ \\
\hline NAL & $146(65,8)$ & $5(2,2)$ & $71(32,0)$ & $69(69,7)$ & $1(1,0)$ & $29(29,3)$ & $77(62,7)$ & $4(3,3)$ & $42(34,1)$ \\
\hline CIP & $193(86,9)$ & $2(0,9)$ & $27(12,1)$ & $87(87,9)$ & $1(1,0)$ & $11(11,1)$ & $106(86,2)$ & $1(0,8)$ & $16(13,0)$ \\
\hline $\mathrm{CHL}$ & $187(84,2)$ & -- & $35(15,8)$ & $84(84,8)$ & -- & $15(15,2)$ & $103(83,7)$ & -- & $20(16,3)$ \\
\hline GEN & $197(88,7)$ & $2(0,9)$ & $23(10,3)$ & $91(91,9)$ & $1(1,0)$ & $7(7,1)$ & $106(86,2)$ & $1(0,8)$ & $16(13,0)$ \\
\hline NIT & $200(90,1)$ & $7(3,2)$ & $12(5,4)$ & $87(86,9)$ & $7(7,1)$ & $5(5,0)$ & $116(94,3)$ & -- & $7(5,7)$ \\
\hline AMC & $189(85,1)$ & $23(10,4)$ & $10(4,5)$ & $85(85,9)$ & $10(10,1)$ & $4(4,0)$ & $104(84,5)$ & $13(10,6)$ & $6(4,9)$ \\
\hline CTX & $212(95,5)$ & $7(3,2)$ & $3(1,4)$ & $96(97,0)$ & $2(2,0)$ & $1(1,0)$ & $116(94,3)$ & $5(4,1)$ & $2(1,6)$ \\
\hline $\mathrm{CAZ}^{*}$ & $216(97,7)$ & $3(1,3)$ & $2(0,9)$ & $97(98,0)$ & $2(2,0)$ & -- & $119(97,6)$ & $1(0,8)$ & $2(1,6)$ \\
\hline
\end{tabular}

AMP: ampicilina; SXT: cotrimoxazol; TET: tetraciclina; NAL: ác. nalidíxico; CIP: ciprofloxacino; CHL: cloranfenicol; GEN: gentamicina; NIT: nitrofurantoina; AMC:amoxicilina más ácido clavulánico; CTX: cefotaxima; CAZ: ceftazidima; A.A: agente antimicrobiano.

S: sensible; I: intermedio; R: resistente

*Datos de 221 aislados. † p<0,05 por la comparación entre niños 0-6 meses y niños 7-12 meses de edad.

Al realizar el análisis por edad, se observó que los niveles de resistencia a SXT eran significativamente mayores en el grupo de niños mayores de 6 a 12 meses, comparado con el grupo de menor edad $(p<0,05)$.

En el caso de la azitromicina se agrupó por el tamaño del diámetro de inhibición. En trece aislados $(6,3 \%)$ no se observó halo, mientras que en diez $(5,3 \%)$ y cuarentidós $(20,3 \%)$ aislados mostraban halo menor de $10 \mathrm{~mm}$ o comprendido entre 11 a $15 \mathrm{~mm}$ respectivamente. El resto de aislados analizados comprendían diámetros entre 16 y $20 \mathrm{~mm}(61 / 207,28 \%)$ o mayor que $20 \mathrm{~mm}$ (90/207, 41,3\%).

Cabe destacar los elevados niveles de resistencia a NAL (32,0\%), así como, en menor número, a CIP $(12,2 \%)$. La presencia de resistencia a NAL pero sensibilidad o resistencia intermedia a CIP fue detectada en cuarentidós $(18,9 \%)$ y dos $(0,9 \%)$ cepas

Tabla 2. Fenotipos de resistencia a quinolonas encontrado en las cepas comensales.

\begin{tabular}{|c|c|c|}
\hline \multirow[b]{2}{*}{ Fenotipo } & \multicolumn{2}{|c|}{ Aislados } \\
\hline & N. ${ }^{\circ}$ & $\%$ \\
\hline $\mathrm{Nal}^{\mathrm{S}} \mathrm{Cip}^{\mathrm{s}}$ & 146 & 65,8 \\
\hline Nal' Cips & 5 & 2,2 \\
\hline $\mathrm{Nal}^{\mathrm{R}} \mathrm{Cip}^{\mathrm{S}}$ & 42 & 18,9 \\
\hline $\mathrm{Nal}^{\mathrm{R}} \mathrm{Cip}{ }^{\prime}$ & 2 & 0,9 \\
\hline $\mathrm{Nal}^{\mathrm{R}} \mathrm{Cip}^{\mathrm{R}}$ & 27 & 12,2 \\
\hline TOTAL & 222 & 100,0 \\
\hline
\end{tabular}

Nal: ácido nalidíxico, Cip: ciprofloxacino

En superíndice, R: resistente, I: resistencia intermedia, S: sensible. respectivamente. Se encontraron cinco (2,3\%) aislados que presentaban resistencia intermedia a NAL. No se encontró ningún aislado que presentara resistencia a CIP y fuese sensible o con resistencia intermedia a NAL (Tabla 2).

Se detectó multirresistencia en noventiún aislados (91/222, 41,0\%). Además, se encontraron cepas que presentaban resistencia intermedia a diferentes antimicrobianos. De estos aislados, la mayoría exhibían resistencia intermedia a AMC.

Se observaron un total de 81 perfiles de resistencia, los encontrados con mayor frecuencia fueron los "pansusceptible" (sensibles a todos los antimicrobianos evaluados; Tabla 3). El resto de perfiles de resistencia encontrados tenían menos de diez aislados cada uno ( $<5 \%$ de todos los aislados).

Tabla 3. Perfiles principales de sensibilidad a antimicrobianos.

\begin{tabular}{|c|c|c|}
\hline Perfiles & $\mathrm{N}^{\text {o* }}$ & $\%$ \\
\hline Sensible a todos A.A & 49 & 22 \\
\hline AMPR $^{R} S X T^{R} T_{E} T^{R}$ & 16 & 7,2 \\
\hline $\mathrm{AMP}^{\mathrm{R}}$ & 14 & 6,3 \\
\hline $\mathrm{AMP}^{\mathrm{R}} \mathrm{S} X T^{\mathrm{R}}$ & 13 & 5,9 \\
\hline 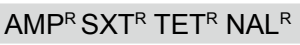 & 13 & 5,9 \\
\hline Otros $^{1}$ & 117 & 52,7 \\
\hline
\end{tabular}

* Número de cepas aisladas.

${ }^{1}$ Setenta y seis perfiles de sensibilidad que presentaban porcentajes menores de $5 \%$ entre todos los aislados.

A.A: agente antimicrobiano; AMP: ampicilina; SXT: cotrimoxazol; TET: tetraciclina; NAL: ác. nalidíxico.

S: sensible; I: intermedio; R: resistente 


\section{DISCUSIÓN}

El uso de las quinolonas, a pesar de demostrarse su seguridad en niños ${ }^{(3)}$, se limita a situaciones y usos específicos, en Perú no es una excepción. De este hecho, y debido a la falta de datos de consumo de antimicrobianos, se infiere que los elevados niveles de resistencia a quinolonas, descritos en las cepas de este estudio, no son por la presión directa después de un tratamiento, sino a razones externas.

En un estudio reciente, realizado en una comunidad alejada de la Amazonía peruana, donde el uso de antimicrobianos es limitado, no se encontraron microorganismos aislados de $E$. coli resistentes a quinolonas, ni en niños ni en adultos ${ }^{(8)}$. Este hecho sugiere que la resistencia a quinolonas, descrita en el presente estudio, se debería a la fuerte influencia de la exposición a los antimicrobianos de manera persistente. Así, la resistencia a quinolonas se podría considerar como un buen marcador de la exposición a los antimicrobianos.

Los presentes resultados sugieren un gran uso de estos antimicrobianos en la comunidad. Este hecho podría haber originado la selección de aislados resistentes a quinolonas, los cuales habrían sido adquiridos por los niños e incorporados como parte de su microbiota intestinal comensal. Por tanto, probablemente las $E$. coli comensales resistentes a quinolonas observadas, fueron adquiridas directamente del entorno familiar. Existen estudios previos en otra zona periurbana de Lima que muestran aislados de $E$. coli de adultos voluntarios sanos, que presentaban niveles de resistencia a ciprofloxacino superiores al $30 \%$ (9). Además, actualmente se puede comprar antimicrobianos sin prescripción, de donde se puede derivar con facilidad a tratamientos y posologías incorrectas, siendo ambos factores favorecedores para la aparición y selección de resistencias a antimicrobianos. Por último, debemos señalar los elevados niveles de resistencia a quinolonas que se han detectado en microorganismos procedentes de piscifactorías ${ }^{(10)}$. Estos datos reflejan los elevados niveles de uso de las quinolonas y sugieren un rol relevante de la cadena alimentaria en la diseminación de la resistencia en el área.

El uso inapropiado de estos antimicrobianos y la aparición de resistencias, no está limitado a microorganismos comensales sino que también es extensible a microorganismos patogénicos, resultando en una pérdida de su potencial para tratar distintas infecciones. Dando soporte a estas afirmaciones, se han descrito altos niveles de resistencia a quinolonas en E. coli diaerrogénicas en la misma área ${ }^{(11,12)}$.
Es necesario destacar los elevados niveles de resistencia encontrados en estas cepas comensales y así como en diaerrogénicas de la misma cohorte ${ }^{(12)}$, frente a otros antimicrobianos de uso frecuente en pediatría, como son AMP, SXT, entre otros, lo cual sugiere la necesidad urgente de búsqueda de tratamientos alternativos eficaces. Por el momento otros antimicrobianos, como el caso de la azitromicina, se han añadido a la lista para tratar la diarrea. Desafortunadamente, el presente estudio también muestra aislados resistentes a AZM en la zona.

Estos datos elevados de resistencias a antimicrobianos se podrían relacionar con datos de un estudio realizado en ocho países de América Latina, en el que se describe la evolución del consumo de antimicrobianos en la población, entre 1997 y 2007, donde se observa a Perú como uno de los países donde se había incrementado el consumo de antimicrobianos ${ }^{(13)}$.

En el resto de antimicrobianos analizados, no pertenecientes al grupo de las quinolonas, la resistencia sería atribuible a presión directa a la que están sometidas las E. coli comensales. Los niveles significativamente más elevados de resistencia a SXT en niños entre 7 y 12 meses apoya esta posibilidad, dado que este es uno de los antimicrobianos más extensamente usados para tratar infecciones infantiles. Sin embargo, los mecanismos de resistencia más frecuentemente descritos frente a estos antimicrobianos están localizados en elementos móviles ${ }^{(14)}$ y, por tanto, no se debe descartar un origen exógeno de la adquisición de mecanismos moleculares de resistencia.

Previos informes nos muestran cierto grado de clonalidad en cepas de E.coli comensales, realizados en pequeñas comunidades aisladas. A pesar de que los perfiles de resistencia a antimicrobianos no son un buen marcador de las relaciones clonales, la elevada variedad de perfiles encontrados podrían reflejar el bajo grado de clonalidad de las cepas de $E$. coli comensales estudiadas. Una heterogeneidad de perfiles de resistencia similar han sido informados previamente en otros estudios de cepas no patogénicas de dicha bacteria ${ }^{(14,15)}$.

Al ser este estudio de una región limitada de Lima, podría ser que estos datos no fueran representativos de otras regiones del país.

Como conclusión, este informe muestra los altos niveles de resistencia a antimicrobianos, especialmente en quinolonas en niños menores de 1 año, en Lima. Estos datos pueden sugerir un elevado consumo de antimicrobianos en la comunidad, así como nos muestran el riesgo inminente de pérdida de eficiencia 
de las quinolonas para tratar enfermedades diarreicas $\mathrm{u}$ otras infecciones, se propone la necesidad de buscar nuevas alternativas de tratamiento.

\section{AGRADECIMIENTOS}

Agradecemos a Laura Puyol por su apoyo en la logística del laboratorio.

\section{Contribuciones de autoría}

AIG, CFL, SM, JR participaron en la concepción y diseño del estudio; AIG, CFL, MM, TJO y JR en el aporte de pacientes o material de estudio; CFL, TJO y JR en la obtención del financiamiento; TJO, SM, MJP, JR, y LJdV participaron el análisis e interpretación de los datos. Todos los autores participaron de la recolección de resultados, revisión crítica del manuscrito, aprobación de su versión final.

\section{Conflictos de interés}

Los autores declaran no tener ningún conflicto de interés.

\section{Fuentes de financiamiento}

Este trabajo fue parcialmente financiado por Agència Catalana de Cooperació al Desenvolupament proyecto U2006 (LJdV), Centre de Cooperació per al Desenvolupament - Universitat Politècnica de Catalunya (LJdV), Agencia Española de Cooperación Internacional al Desarrollo proyectos numero A/4892/06 (LJdV), D/019499/08 y D/024648/09 (JR), Fogarty International Center, National Institute of Health, USA, proyecto 1K01TW007405 (TJO) Sanofi Pasteur y fondos de investigación del Dr. Lanata, Instituto de Investigación Nutricional, Lima, Perú. La investigación de JR es financiado por el proyecto CP05/0130 del FIS (Fondo de Investigaciones Sanitarias, España).

\section{REFERENCIAS BIBLIOGRÁFICAS}

1. Organización Mundial de la Salud. Estrategia mundial de la OMS para contener la resistencia a los antimicrobianos. Ginebra: OMS; 2001.

2. Ruiz J. Mechanisms of resistance to quinolones: target alterations, decreased accumulation and DNA gyrase protection. J Antimicrob Chemother. 2003;51(5):1109-17.

3. Murray TS, Baltimore RS. Pediatric uses of fluoroquinolone antibiotics. Pediatric Ann. 2007;36(6):336-42.

4. Strahilevitz J, Jacoby GA, Hooper DC, Robicsek A. Plasmidmediated quinolone resistance: a multifaceted threat. Clin Microbiol Rev. 2009;22(4):664-89.
5. Tannock GW. Normal microflora. An introduction to microbes inhabiting the human body. London: Ed-Chapman and Hall; 1995.

6. Ochoa TJ, Ecker L, Barletta F, Mispireta ML, Gil Al, Contreras $\mathrm{C}$, et al. Age-related susceptibility to infection with diarrheagenic Escherichia coli among Infants from Periurban Areas in Lima, Peru. Clin Infect Dis. 2009;49(11):1694-702.

7. Clinical and Laboratory Standards Institute. Performance standards for antimicrobial susceptibility testing. Fifteenth informational supplement. Wayne, PA: CLSI; 2005.

8. Bartoloni A, Pallecchi L, Rodriguez H, Fernandez C, Mantella A, Bartalesi F, et al. Antibiotic resistance in a very remote Amazonas community. Internat J Antimicrob agents. 2009;33(2):125-9.

9. Nys S, Okeke IN, Kariuki S, Dinant GJ, Driessen C, Stobberingh EE. Antibiotic resistance of faecal Escherichia coli from healthy volunteers from eight developing countries. J Antimicrob Chemother. 2004;54(5):952-5.

10. Deza J, Sierralta V, León J, Mateo E. Sensibilidad antibiótica in vitro de Yersinia ruckeri aisladas de Oncorhynchus "trucha arcoiris" de piscigranja del centro del Perú. XVIII Reunión Científica del ICBAR. Universidad Nacional Mayor de San Marcos, Facultad de Ciencias Biológicas. Lima, Perú. 2009.

11. Fàbrega A, Sánchez-Céspedes J, Soto S, Vila J. Quinolone resistance in the food chain. Int $\mathrm{J}$ Antimicrob Agents. 2008;31(4):307-15

12. Ochoa TJ, Ruiz J, Molina M, Del Valle LJ, Vargas M, Gil Al, et al. High frequency of antimicrobial drug resistance of diarrheagenic Escherichia coli in infants in Peru. Am J Trop Med Hyg. 2009;81(2):296-301.

13. Wirtz VJ, Dreser A, Gonzales R. Trends in antibiotic utilization in eight Latin American countries, 1997-2007. Rev Panam Salud Publica. 2010;27(3):219-25.

14. Pallecchi L, Lucchetti C, Bartoloni A, Bartalesi F, Mantella A, $\mathrm{Gamboa} \mathrm{H}$, et al. Population structure and resistance genes in antibiotic-resistant bacteria from remote community with minimal antibiotic exposure. Antimicrob Agents Chemother. 2007;51(4):1179-84.

15. Domínguez E, Zarazaga M, Sáenz $\mathrm{Y}$, Briñas L, Torres $\mathrm{C}$. Mechanisms of antibiotic resistance in escherichia coli isolates obtained from healthy children in Spain. Microb Drug Resist. 2002;8(4):321-7.

Correspondencia: Joaquim Ruiz

Dirección: Ed. CEK, pl1, C/ Rosselló 153, 08036, Barcelona, España.

Teléfono: (34) 932275400 ext 4547, fax: (34) 932279853

Correo electrónico: joruiz@clinic.ub.es 